\title{
Creencias de los estudiantes de Educación Secundaria Obligatoria sobre la diversidad funcional
}

\section{Beliefs of Compulsory Secondary Education students on functional diversity}

\author{
María Martínez Galiana ${ }^{1}$ \\ m.martinezgaliana@gmail.com \\ Mángeles Gomariz Vicente \\ magovi@um.es \\ Antonia Cascales Martínez \\ antonia.cascales@um.es \\ Universidad de Murcia, España
}

\section{Resumen:}

En el presente trabajo se pretende dar a conocer las creencias que tienen los estudiantes de Educación Secundaria Obligatoria acerca de la diversidad funcional, a partir de un estudio principalmente de carácter descriptivo realizado mediante la técnica de encuesta aplicada a 442 adolescentes de entre doce y dieciocho años, tanto de la Región de Murcia como de la Comunidad Valenciana. Los resultados muestran que los adolescentes que han crecido en una sociedad considerada inclusiva tienen una opinión respetuosa y beneficiosa respecto a la integración de personas con diversidad funcional en la sociedad, si bien esta opinión está vinculada, entre otros aspectos, al centro en el que estudie o a la relación con la discapacidad.

\section{Palabras clave:}

Necesidades educativas; adolescentes; diversidad funcional; inclusión educativa.

\begin{abstract}
:
This article presents Compulsory Secondary Education students' beliefs on functional diversity. The study adopted a mainly descriptive research design based on the administration of surveys to 442 adolescents between the ages of twelve and eighteen, both in the Region of Murcia and the Valencian Community. The results show that these adolescents, in having grown up in a society considered inclusive, have respectful and positive opinions regarding the integration in society of people with functional diversity. These opinions are linked, among other aspects, to the secondary school students attend and to their relationship with disability.
\end{abstract}

\section{Keywords:}

Special education needs; adolescents; functional diversity; inclusive education.

1 Dirección para correspondencia (correspondence address):

María Martínez Galiana. Departamento Métodos de Investigación y Diagnóstico en Educación. Universidad de Murcia. Facultad de Educación, Campus de Espinardo, 30100 Murcia (España). 
Creencias de los estudiantes de Educación Secundaria Obligatoria sobre la diversidad funcional

María Martínez Galiana, Ma Ángeles Gomariz Vicente y Antonia Cascales Martínez

\section{Résumé:}

Dans le présent travail, nous avons l'intention de présenter les convictions des élèves de I'enseignement secondaire obligatoire sur la diversité fonctionnelle, à partir d'une étude principalement à caractère descriptif réalisée au moyen de la technique d'enquête appliquée à 442 adolescents âgés de 12 à 18 ans, tant dans la région de Murcie que dans la communauté valencienne. Les résultats montrent que les adolescents qui ont grandi dans une société considérée comme inclusive ont une opinion respectueuse et bénéfique de l'intégration des personnes ayant une diversité fonctionnelle dans la société, bien que cette opinion soit liée, entre autres, au centre dans lequel ils ont étudié ou à la relation avec le handicap.

\section{Mots-clés:}

Besoins éducatifs; adolescents; diversité fonctionnelle; inclusion scolaire.

Fecha de recepción: 18-01-2018

Fecha de aceptación: 11-06-2019

\section{Introducción}

La escuela inclusiva es aquella en la cual pueden aprender junto alumnado diferente, personas con unas características y unas capacidades propias. Ello tiene lugar en centros ordinarios a los que puede (y debe) asistir todo el alumnado, comprendiendo a aquellos que tienen diversidad funcional, pertenecen a culturas o etnias diferentes, se encuentran en situaciones de pobreza o en cualquier otra situación que pueda dificultar su proceso de escolarización (Forlin, Earle, Loreman y Sharma, 2011).

En España, este término ha evolucionado hasta llegar a la actual Ley Orgánica 8/2013, de 9 de diciembre, para la Mejora de la Calidad Educativa (LOMCE). Dicha ley modifica los apartados 1 y 2 del art. 71, añade el art. 76 sobre altas capacidades y el art 79.bis y, consolidando los aspectos relacionados con la atención a la diversidad defendidos en la Ley Orgánica 2/2006, de 3 de mayo, de Educación (LOE, 2006), con el fin de regular la respuesta educativa a la diversidad, a su evaluación y su intervención tanto con los alumnos con necesidades educativas especiales (ACNEE) como con los alumnos con necesidades específicas de apoyo educativo (ACNEAE).

Este concepto, no está igualmente interiorizado en la sociedad, sino que se continúa menospreciando las capacidades de las personas con diversidad funcional. Echeita $(2004,2014)$ sostiene que la sociedad tiende a emplear un "patrón normativo" donde cualquier diferencia puede 
ser considerada como una carencia, una anormalidad o un desvío, y las intervenciones que se realizan para minimizar estas desigualdades van dirigidas, sobre todo, a convertir los rasgos que más se alejan del patrón normativo en cualidades lo más ordinarias posible. Es por ello que, de acuerdo con Belmonte y García-Sanz (2013), actualmente la integración de estas personas no se ha interiorizado, pese a los cambios positivos que se están produciendo a favor de la no-discriminación.

\section{Marco teórico}

\section{De la Educación Especial a la Inclusión Educativa en la normativa}

La escolarización de ACNEE debida a una discapacidad o como consecuencia de un trastorno grave de conducta ha ido evolucionando con el paso del tiempo. Hasta finales del siglo XIX se llevaba a cabo en instituciones médicas, es a partir del s. XX cuando comenzaron a cuestionarse estas prácticas como pedagógicas. Fue en ese momento cuando surgió un movimiento de cambio conocido como Era de la Normalización, con el objetivo de poner fin a la educación exclusiva que se había estado llevando a cabo hasta entonces.

En España con la promulgación de la Constitución de 1978, se garantiza la igualdad de todos los españoles y su derecho a la educación, independientemente de sus características personales. La Ley 13/1982, de Integración Social de los Minusválidos (LISMI), fomentaba la desaparición progresiva de la Educación Especial como un subsistema de la educación ordinaria, a partir de la defensa de los principios de normalización, integración, sectorización e individualización (González, 2012). Más tarde, la Ley Orgánica 1/1990, de 3 de octubre, de Ordenación General del Sistema Educativo (LOGSE), supuso una transformación del sistema educativo, pues aseguraba una educación normalizada e inclusiva en la que los centros ordinarios debían contar con los recursos específicos para atender a la diversidad del alumnado en un entorno lo menos restrictivo posible.

La LOE (2006), estableció una serie de propuestas y principios que continuaron amparándose en la LOMCE (2013). En ella, se delimita a los centros ordinarios como las instituciones más efectivas para llevar a cabo la inclusión educativa, los cuales se debían adecuar a la diversidad 
del alumnado para favorecer su proceso de enseñanza-aprendizaje. Para ello es necesario valorar las diferencias individuales de cada uno de sus alumnos y alumnas, considerándolas no como un hándicap que dificulta el proceso de enseñanza-aprendizaje, sino como una oportunidad que facilitará el desarrollo integral de todos los discentes (González, 2012).

En síntesis, toda esta normativa nos señala que en España se ha producido una evolución conceptual, inclinándose por la inclusión y redimensionando el significado de esta política en la práctica.

\section{La actitud ante la inclusión educativa y la diversidad funcional}

Se puede afirmar que el Informe Warnock (Aguilar Montero, 1991) marcó un punto de inflexión en el concepto de normalización, en la medida que aceptaba persona con necesidades educativas especiales (NEE) como es, con sus necesidades, mismos derechos que los demás y garantizándole los servicios para que pueda desarrollar al máximo sus potencialidades. Ello supone acabar con los términos peyorativos que se habían estado empleando hacia las personas con discapacidad, los cuales hacían referencia a las dificultades que podía presentar un niño o niña (Jiménez y Huete, 2009).

Si bien debemos diferenciar la discapacidad de la NEE. La discapacidad está vinculada a la dificultad que la persona tenga para acceder a todos los ámbitos relacionados con la Educación. En este sentido el término de atención a la diversidad que, de acuerdo con Arnáiz, Guirao y Garrido (2007), describía la modalidad educativa inclusiva que fomentaba el desarrollo integral de todo el alumnado en una única escuela a la que todos los individuos de la sociedad debían asistir. Finalmente, y teniendo en cuenta al Colectivo loé (2013), en el año 2005 se propuso un nuevo vocablo para dirigirse a las personas con algún tipo de discapacidad. Surgió así el término de diversidad funcional, que afirmaba que todas las personas tenían unas capacidades diversas que se debían aceptar desde el inicio con el objetivo de fomentar la igualdad en todos los aspectos de sus vidas. El término diversidad hace referencia a las diferencias existentes entre todas las personas, y no es exclusivo de aquellas personas que tienen alguna discapacidad (Dussan, 2011).

De acuerdo con Arnáiz (2003), vivimos en una sociedad en la que la exclusión y la marginación debido a condiciones personales, sociales o culturales es uno de los problemas más relevantes. 
Entendiendo la actitud como una "disposición mental que influye sobre las respuestas del individuo a los estímulos y situaciones con los que se relaciona" (Mampaso, 2004, p.180), se hace necesario analizar las conductas que la discapacidad origina en el resto de individuos de la sociedad, las cuales han ido evolucionando de forma positiva al incorporar la inclusión al modelo educativo. Sin embargo, nos planteamos en qué medida los estudiantes que han crecido en una sociedad inclusiva continúan teniendo actitudes discriminatorias hacia este colectivo; si verdaderamente, se están cumpliendo los objetivos propuestos o, por el contrario, no se han eliminado las barreras que les permiten incluirse de forma eficaz (Muratori, Guntín y Delfino, 2010).

En esta línea, Muntaner (1998) afirma que:

Cuando la sociedad aprende a respetar las diferencias y no centra su atención en las deficiencias de las personas, sino en sus capacidades, y cuando aprende también a valorarlos no por su apariencia, sino por su valía real, el problema comenzará a desaparecer (p.11).

Por tanto, la inclusión educativa va más allá de ofrecer una serie de recursos, sino que, tal como indica González-Gil (2011), se trata de fomentar la pertenencia a un grupo, de sentirse querido y formar parte de él; hecho que involucra las actitudes y pensamientos de todos los individuos de la comunidad. Igualmente, según Pineda (2017), se trata de ayudarles a creer en sí mismos y en sus posibilidades, para lo que es necesario que se promueva la normalización a nivel familiar, educativo y social a través de la transmisión de valores como el respeto o la tolerancia hacia las personas con diversidad funcional (Luque y Luque-Rojas, 2012).

La revisión de la literatura especializada, nos indica que existen factores personales y ambientales que intervienen de forma significativa para definir una opinión sobre la discapacidad, entre los que destaca el conocimiento de la misma y la integración de los discapacitados en la sociedad (Aguado, Alcedo y Arias, 2008; Aguado, Flórez y Alcedo, 2004; Santana, 2010). Bausela (2009) realizó una investigación con el objetivo de conocer las actitudes hacia la discapacidad de un grupo de estudiantes de psicología concluyendo que las actitudes son una de las variables más relevantes para facilitar la integración de las personas con discapacidad. En esta misma línea, García y Hernández (2011), nos 
señalan que aquellas personas que tienen un familiar con discapacidad, que son más jóvenes y que viven en zonas urbanas muestran actitudes positivas hacia la discapacidad, sin embargo, el género no se considera vinculante para definir estas actitudes.

A raíz de lo expuesto, nos planteamos explorar las creencias y pensamientos de los adolescentes nacidos durante la era de la inclusión educativa, acerca de sus iguales con diversidad funcional, partiendo de cuestiones tan relevantes como: ¿En qué medida, determinan la opinión que pueda desarrollar el alumnado de estas edades sobre las personas con diversidad funcional sus aspectos sociodemográficos como la edad, el curso educativo y el tipo de escuela? ¿Qué tipo de relación mantienen con la discapacidad?

\section{Marco empírico}

\subsection{Objetivos}

A partir de la revisión teórica, nos proponemos como objetivo general de este estudio conocer la opinión de los adolescentes sobre la discapacidad o diversidad funcional. Este propósito general se articula, a su vez, en dos objetivos específicos que lo complementan y contribuyen a la consecución del mismo:

1. Conocer la opinión de los adolescentes de entre 12 y 18 años sobre la discapacidad o diversidad funcional.

2. Analizar la influencia de las variables sociodemográficas (género, edad, curso que se está estudiando, tipo de centro al que asiste, relación con la discapacidad) en la opinión que tienen los adolescentes sobre la diversidad funcional.

\subsection{Diseño metodológico}

Se trata de un estudio de carácter no experimental, con un enfoque de corte descriptivo, el cual pretende aportar información rigurosa para realizar un diagnóstico de la realidad en la que se encuentran los estudiantes de Educación Secundaria (Cubo, Martín y Ramos, 2011).

Para la recogida de la información, se ha utilizado un instrumento tipo encuesta, diseñado ad hoc, que ha permitido obtener información 
detallada de 470 alumnos y alumnas de entre 12 y 18 años. La propia naturaleza del instrumento que parte de las inquietudes de los autores tras consultar la bibliografía estudiada aconsejaba su validación con una estrategia de validez entre jueces de carácter cualitativo, para lo cual se contó con cinco expertos: dos orientadores de un Equipo de Orientación Educativa y Psicopedagógica, una doctora en pedagogía y una orientadora de un Instituto de Educación Secundaria. Tras dicha validación se realizaron mínimas aportaciones que fueron incorporadas junto con algunas modificaciones de redacción a la versión final del instrumento.

La encuesta está compuesta por un total de 44 preguntas, tanto abiertas como cerradas. Las diez primeras están dirigidas a la identificación del alumno en cuestión, por lo que se les ha preguntado acerca de la edad, el género, el curso y el tipo de centro en el que están matriculados, su relación más directa con la diversidad funcional y si reciben apoyo educativo. Las dos siguientes, son preguntas abiertas en las que los participantes han de explicar qué es la discapacidad para ellos y qué entienden por el término de diversidad funcional; y una última cuestión de opciones múltiples nos permite conocer qué piensan los participantes sobre qué es una discapacidad. La segunda parte de la encuesta consta de 31 preguntas. De ellas, las trece primeras preguntas están elaboradas a partir de una escala Likert, compuesta por ítems que han permitido conocer su relación con la discapacidad y cuyas respuestas están categorizadas del 1 al 4, siendo (1) Nunca, (2) Casi nunca, (3) Casi siempre y (4) Siempre. Sin embargo, aunque en las siguientes dieciocho cuestiones también se ha empleado la misma escala, sus ítems recogen información acerca de su opinión frente a la diversidad funcional, por lo que sus respuestas están numeradas del 1 al 4, siendo (1) Totalmente en desacuerdo, (2) En desacuerdo, (3) De acuerdo y (4) Totalmente de acuerdo.

Además, los ítems que forman la segunda parte del instrumento (ítems 14 a 44) pueden dividirse en seis dimensiones, las cuales quedan reflejadas en la Tabla 1. 
Creencias de los estudiantes de Educación Secundaria Obligatoria sobre la diversidad funcional

María Martínez Galiana, Ma Ángeles Gomariz Vicente y Antonia Cascales Martínez

Tabla 1

Dimensiones evaluadas en el instrumento de recogida de información

\begin{tabular}{cll}
\hline $\begin{array}{c}\text { Número } \\
\text { de ítems }\end{array}$ & \multicolumn{1}{c}{ Dimensión } & \multicolumn{1}{c}{ Descripción } \\
\hline 8 & $\begin{array}{l}\text { 1. Relación con } \\
\text { la discapacidad }\end{array}$ & $\begin{array}{l}\text { Evalúa la relación que los estudiantes de Educa- } \\
\text { ción Secundaria Obligatoria mantienen con las } \\
\text { personas con discapacidad. }\end{array}$ \\
\hline 5 & $\begin{array}{l}\text { 2. Respeto y } \\
\text { tolerancia }\end{array}$ & $\begin{array}{l}\text { Valora la actitud de los adolescentes cuando ob- } \\
\text { servan actitudes inadecuadas frente a personas } \\
\text { con discapacidad. }\end{array}$ \\
\hline 3 & $\begin{array}{l}\text { 3. Adquisición de } \\
\text { conocimientos }\end{array}$ & $\begin{array}{l}\text { Estima el grado en el que los adolescentes han } \\
\text { adquirido información de diversos medios acer- } \\
\text { ca de la diversidad funcional. }\end{array}$ \\
\hline 3 & $\begin{array}{l}\text { 4. Derechos } \\
\text { habilidades }\end{array}$ & $\begin{array}{l}\text { Evalúa si los adolescentes piensan que tienen las } \\
\text { mismas oportunidades y los mismos derechos. }\end{array}$ \\
\hline 4 & $\begin{array}{l}\text { 6. Adaptación } \\
\text { y utilización de } \\
\text { recursos }\end{array}$ & $\begin{array}{l}\text { Determina si los adolescentes piensan que la so- } \\
\text { ciedad infravalora las capacidades y habilidades } \\
\text { de las personas con diversidad funcional. }\end{array}$ \\
\hline & $\begin{array}{l}\text { Menciona el grado de recursos, adaptaciones y } \\
\text { apoyos con los que se cuenta para ayudar a estas } \\
\text { personas }\end{array}$ \\
\hline
\end{tabular}

El nivel de fiabilidad de la encuesta fue analizado mediante el alfa de Cronbach (O'Dwyer y Bernauer, 2014), obteniendo una puntuación global de 0.723 . Este resultado indica una consistencia interna respetable (De Vellis, 2003).

\subsection{Participantes}

En este estudio se ha empleado un muestreo deliberado cuya muestra invitada y aceptante es de 470 estudiantes, aunque finalmente la muestra final ha quedado definida por 442 discentes de entre 12 y 18 años. De ellos, se ha observado una mayor participación en el alumnado de 14 (25.1\%) y de 15 años (22.9\%); así como muy poca de 17 (3.8\%) y 18 años (0.5\%). Más de la mitad de los encuestados son mujeres (53.6\%), mientras que un $46.2 \%$ son hombres. $Y$ de todos ellos, un $67 \%$ estudian en la provincia de Alicante, mientras que el $32.6 \%$ restante estudia en la Región de Murcia. En cuanto al curso en el que están matriculados, la mayoría cursaban segundo de Educación Secundaria Obligatoria (ESO) 
(30.8\%) y un $26.2 \%$ lo hacía en primer curso, aunque también participaron alumnos de otros cursos. Un 83\% asiste a centros públicos, mientras que el resto lo hace en centros concertados. Y, de ellos, más de la mitad no recibe ningún tipo de apoyo educativo (59.5\%), frente al $34.9 \%$ que sí lo recibe. La mayoría de los participantes $(60.9 \%)$ ha compartido aula en algún momento de su escolarización con iguales con discapacidad o tiene algún conocido que la presenta (68.8\%). Finalmente, un $96.6 \%$ de la muestra afirma no tener ninguna diversidad funcional, ni tampoco familiares que la posean en un $67.9 \%$ de los casos.

\subsection{Análisis de datos}

El análisis cuantitativo de la información obtenida se ha realizado mediante el paquete estadístico SPSS Statistic (versión 19). Para calcular y contrastar las medias se han empleado dos pruebas de estadística inferencial no paramétrica, como son la prueba $U$ Mann Whitney para comparar variables en dos muestras independientes, y la técnica de KruskalWallis para contrastar más de dos grupos, con el fin de conocer entre qué grupos hay diferencias estadísticamente significativas.

\section{Resultados y discusión}

Los resultados obtenidos se van a estructurar de acuerdo con los objetivos propuestos al inicio del estudio.

Objetivo 1: Conocer la opinión de los adolescentes de entre 12 y 18 años sobre la discapacidad o diversidad funcional.

En primer lugar, se ha preguntado a los estudiantes de ESO acerca de los conocimientos previos sobre el tema:

Qué es la discapacidad para ti

La Tabla 2 refleja la consideración de la discapacidad como un problema que afecta a nivel físico, psíquico o sensorial, lo que dificulta el correcto funcionamiento de los órganos (27.15\%). Asimismo, también queda reflejada la consideración de la diversidad funcional como un 
obstáculo que limita las acciones cotidianas de estas personas para ser autónomos (24.21\%), o la contemplación de la discapacidad como una enfermedad (14.03\%). Por ello, puede afirmarse que la discapacidad es percibida a partir de perspectivas que destacan los aspectos negativos de presentarla. Tan solo un $4.07 \%$ de los encuestados la explican destacando algún aspecto positivo, afirmando que la única discapacidad son aquellas personas que tienen una mala actitud hacia las personas con discapacidad (23\%); es un hándicap, aunque con esfuerzo se pueden lograr las mismas cosas y se puede hacer lo mismo que cualquier otra persona (3.39\%); o una característica especial que no se considera un inconveniente $(.45 \%)$.

Tabla 2

Definiciones de la discapacidad según los estudiantes de ESO. Estadística descriptiva

\begin{tabular}{ll}
\hline Respuesta & $\%$ \\
\hline
\end{tabular}

Limitación de alguna capacidad que te afecta a la hora de realizar $\quad 8.37$ cualquier actividad.

Obstáculo o dificultad que te impide hacer cosas como el resto de personas.

Problema que afecta a nivel físico, psíquico o sensorial y que afecta al 27.15 correcto funcionamiento de los órganos.

Deficiencia que adquieres al nacer, por lo que es involuntaria y te $\quad 9.95$ afecta a lo largo de tu vida.

La única discapacidad son aquellas personas que tienen una mala

actitud hacia las personas con discapacidad.

Es un hándicap, aunque con esfuerzo se pueden lograr las mismas

cosas y se puede hacer lo mismo que cualquier otra persona.

Es la pérdida de habilidades, lo que les permite no alcanzar el ritmo o

no desarrollar bien las cosas.

Personas que tienen problemas cerebrales: les cuesta aprender, entender bromas o dobles sentidos.

Algo que tienen algunas personas y que les diferencia del resto.

Una característica especial que no se considera un inconveniente.

Cuando las personas entienden el mundo de una forma diferente al resto.

No lo sé.

Personas que no pueden defenderse solas, por lo que necesitan ayuda usualmente.

Es una enfermedad que afecta en las actividades cotidianas y te impi- $\quad 14.03$ de realizarlas.

Personas que no tienen las mismas funciones. 
Creencias de los estudiantes de Educación Secundaria Obligatoria sobre la diversidad funcional María Martínez Galiana, Ma Ángeles Gomariz Vicente y Antonia Cascales Martínez

\begin{tabular}{lc}
\hline Respuesta & $\%$ \\
\hline Gente con otras vidas y con otros órganos. & .23 \\
Gente tonta. & .23 \\
Personas con problemas sociales que no se relacionan correctamente. & .23 \\
Personas que tienen menos oportunidades. & .68 \\
\hline
\end{tabular}

Qué es la diversidad funcional

La Tabla 3 determina que una gran cantidad de adolescentes desconocen el término de diversidad funcional $(71.27 \%)$, debido a su escasa difusión. Sin embargo, el 10.41\% conoce que se trata de términos sinónimos.

Tabla 3

Definiciones de la diversidad funcional según los estudiantes de ESO. Estadística descriptiva

\begin{tabular}{lr}
\hline Respuesta & $\%$ \\
\hline No lo sé. & 71.27 \\
Personas que son diferentes al resto. & 1.36 \\
Es la misma definición que la del término discapacidad. & 10.41 \\
Las cosas que una persona puede o no hacer. & 2.94 \\
No poder mover bien alguna parte del cuerpo. & 3.17 \\
Estar sordo o ciego. & .68 \\
Personas que tienen diferentes funciones. & 1.36 \\
El conjunto de las diferentes discapacidades que existen. & 4.98 \\
Los motivos que te impiden trabajar de forma normal. & .45 \\
La marginación de las personas con discapacidad. & .45 \\
Dificultad para relacionarse con otras personas. & 1.13 \\
Todo lo que sabes acerca de la discapacidad. & .45 \\
El movimiento de las articulaciones. & .45 \\
El conjunto de capacidades que tiene una persona. & .90 \\
\hline
\end{tabular}

La Tabla 4 presenta la media y la desviación típica de cada una de las dimensiones del instrumento nombradas anteriormente, compuestas por las preguntas que van desde la 14 hasta la 44. Se puede apreciar que la dimensión mejor valorada por los participantes es la de adaptaciones y recursos empleados para dar respuesta a la diversidad. Sin embargo, la menos estimada es aquella que describe las capacidades y habilidades de los individuos con discapacidad. 
Creencias de los estudiantes de Educación Secundaria Obligatoria sobre la diversidad funcional

María Martínez Galiana, Ma Ángeles Gomariz Vicente y Antonia Cascales Martínez

Tabla 4

Estadísticos descriptivos de todas las dimensiones de la encuesta

\begin{tabular}{lcccccc}
\hline & Relación & Respeto & $\begin{array}{c}\text { Conoci- } \\
\text { mientos }\end{array}$ & \multicolumn{2}{c}{ Derechos Capacidades Adaptaciones } \\
\hline $\mathrm{N}$ & 442 & 442 & 442 & 442 & 442 & 442 \\
Media & 2.74 & 2.83 & 2.18 & 2.63 & 2.05 & 2.94 \\
Desv. Típ. & .57 & .41 & .71 & .50 & .48 & .60 \\
\hline
\end{tabular}

Seguidamente, se pueden observar los resultados obtenidos organizados en torno a las dimensiones de la encuesta.

\section{Relación con la diversidad funcional}

La Tabla 5 muestra la poca frecuencia con la que los encuestados se relacionan con personas con discapacidad física o intelectual $(41.5 \%$ y $43.4 \%$ ), aunque afirman que casi siempre suelen tener facilidad para relacionarse con ellos (35.9\% y $41.6 \%)$. En la mayoría de los casos $(61.3 \%)$ se asegura no compartir actividades extraescolares con personas con diversidad funcional, aunque sí estarían dispuestos a relacionarse con ellos (68.3\%), a colaborar en actividades de voluntariado para ayudarles (49.3\%) o a aceptar una invitación de parte de estas personas $(61.1 \%)$. Esto significa que, aunque en los centros educativos se fomenta la inclusión, los estudiantes no tienen ningún tipo de relación con sus iguales con discapacidad.

Tabla 5

Estadísticos descriptivos de la dimensión Relación con la diversidad funcional de los alumnos de ESO

\begin{tabular}{lcccc}
\hline Relación con la diversidad funcional & $\begin{array}{c}\text { Nunca } \\
\%\end{array}$ & $\begin{array}{c}\text { Casi } \\
\text { nunca } \\
\%\end{array}$ & $\begin{array}{c}\text { Casi } \\
\text { siempre } \\
\%\end{array}$ & $\begin{array}{c}\text { Siempre } \\
\%\end{array}$ \\
\hline $\begin{array}{l}\text { 14. Me relaciono con personas con disca- } \\
\text { pacidad física. }\end{array}$ & 22.0 & 41.5 & 20.4 & 16.1 \\
$\begin{array}{l}\text { 15. Me relaciono con personas con disca- } \\
\text { pacidad intelectual. }\end{array}$ & 21.6 & 43.4 & 20.7 & 14.3 \\
$\begin{array}{l}\text { 16. Tengo facilidad para relacionarme con } \\
\text { personas con discapacidad física. }\end{array}$ & 15.2 & 18.4 & 35.9 & 30.5 \\
$\begin{array}{l}\text { 17. Tengo facilidad para relacionarme con } \\
\text { personas con discapacidad intelectual. }\end{array}$ & 14.3 & 23.1 & 41.6 & 21.0 \\
\hline
\end{tabular}


Creencias de los estudiantes de Educación Secundaria Obligatoria sobre la diversidad funcional María Martínez Galiana, Ma Ángeles Gomariz Vicente y Antonia Cascales Martínez

\begin{tabular}{lcccc}
\hline Relación con la diversidad funcional & $\begin{array}{c}\text { Nunca } \\
\%\end{array}$ & $\begin{array}{c}\text { Casi } \\
\text { nunca } \\
\%\end{array}$ & $\begin{array}{c}\text { Casi } \\
\text { siempre } \\
\%\end{array}$ & $\begin{array}{c}\text { Siempre } \\
\%\end{array}$ \\
\hline $\begin{array}{l}\text { 18. Comparto actividades extraescolares } \\
\text { con algún compañero con discapacidad. }\end{array}$ & 61.3 & 15.4 & 8.8 & 14.5 \\
$\begin{array}{l}\text { 19. Aunque no me relaciono con perso- } \\
\text { nas con discapacidad, estaría dispuesta o } \\
\text { dispuesto a hacerlo. }\end{array}$ & 5.4 & 7.3 & 19.0 & 68.3 \\
$\begin{array}{l}\text { 20. Colaboraría en actividades de vo- } \\
\text { luntariado para ayudar a personas con } \\
\text { discapacidad. }\end{array}$ & 6.8 & 16.5 & 27.4 & 49.3 \\
$\begin{array}{l}\text { 21. Aceptaría una invitación a un cum- } \\
\text { pleaños o a salir a tomar algo de una }\end{array}$ & 5.0 & 11.3 & 22.6 & 61.1 \\
persona con discapacidad. & & & & \\
\hline
\end{tabular}

Respeto y tolerancia hacia las personas con diversidad funcional

La Tabla 6 revela que los adolescentes suelen tener sensación de malestar al contemplar faltas de respeto hacia la discapacidad (75.1\%), aunque tan solo el $46.2 \%$ afirma defenderlos en este tipo de circunstancias.

Tabla 6

Estadísticos descriptivos de la dimensión Respeto y tolerancia hacia las personas con discapacidad por parte de los estudiantes de ESO

\begin{tabular}{lcccc}
\hline $\begin{array}{l}\text { Respeto y tolerancia hacia las personas con } \\
\text { discapacidad }\end{array}$ & $\begin{array}{c}\text { Nunca } \\
\%\end{array}$ & $\begin{array}{c}\text { Casi } \\
\text { nunca } \\
\%\end{array}$ & $\begin{array}{c}\text { Casi } \\
\text { siempre }\end{array}$ & $\begin{array}{c}\text { Siempre } \\
\%\end{array}$ \\
\hline $\begin{array}{l}\text { 22. Me siento mal ante faltas de respeto } \\
\text { hacia las personas con discapacidad. }\end{array}$ & 5.4 & 5.4 & 14.1 & 75.1 \\
$\begin{array}{l}\text { 23. He defendido a las personas con disca- } \\
\text { pacidad cuando se tienen faltas de respeto }\end{array}$ & 9.3 & 13.8 & 30.8 & 46.2 \\
hacia él o ella. & & & & \\
\hline
\end{tabular}

En cambio, en la Tabla 7 se refleja el desacuerdo de los jóvenes con la forma empleada para nombrar a estas personas (73.6\%), y muestran disconformidad sobre la manera de verlos en sociedad. Además, confirman que no tendrían problema alguno si alguna de sus amistades presentara alguna discapacidad (60.0\%). 
Creencias de los estudiantes de Educación Secundaria Obligatoria sobre la diversidad funcional

María Martínez Galiana, Ma Ángeles Gomariz Vicente y Antonia Cascales Martínez

Tabla 7

Estadísticos descriptivos de la dimensión Respeto y tolerancia hacia las personas con discapacidad por parte de los estudiantes de ESO

\begin{tabular}{lcccc}
\hline $\begin{array}{l}\text { Respeto y tolerancia hacia las } \\
\text { personas con discapacidad }\end{array}$ & $\begin{array}{c}\text { Totalmente } \\
\text { en } \\
\text { desacuerdo } \\
\%\end{array}$ & $\begin{array}{c}\text { En } \\
\text { desacuerdo } \\
\%\end{array}$ & $\begin{array}{c}\text { De } \\
\text { acuerdo } \\
\%\end{array}$ & $\begin{array}{c}\text { Totalmente } \\
\text { de } \\
\text { acuerdo } \\
\%\end{array}$ \\
\hline $\begin{array}{l}\text { 27. La manera por la que } \\
\text { comúnmente se les denomina } \\
\text { (minusválido, retrasado, loco, }\end{array}$ & 73.6 & 11.8 & 4.5 & 10.0 \\
$\begin{array}{l}\text { imbécil...) es respetuosa. } \\
\begin{array}{l}\text { 28. No me importaría que } \\
\text { alguno de mis amigos tuviera } \\
\text { alguna discapacidad. }\end{array}\end{array}$ & 6.6 & 4.5 & 28.9 & 60.0 \\
$\begin{array}{l}\text { 44. Las personas con discapaci- } \\
\text { dad son bien vistas en sociedad. }\end{array}$ & 14.7 & 38.0 & 28.7 & 18.6 \\
\hline
\end{tabular}

La adquisición de los conocimientos acerca de la diversidad funcional

La transmisión de conocimientos acerca de esta temática es limitada, puesto que, de acuerdo con la Tabla 8 , un $35.7 \%$ de los participantes casi nunca han hablado sobre la discapacidad. Asimismo, el $51.1 \%$ asegura no haber asistido nunca a charlas en las que se trate esta materia en las instituciones educativas, ni ha visionado películas ni ha leído libros acerca de la discapacidad casi nunca (29.9\%).

Tabla 8

Estadísticos descriptivos de la dimensión Adquisición de conocimientos respecto a la discapacidad desde la opinión de los alumnos de ESO

\begin{tabular}{lcccc}
\hline $\begin{array}{l}\text { Adquisición de conocimientos acerca de la } \\
\text { diversidad funcional }\end{array}$ & $\begin{array}{c}\text { Nunca } \\
\%\end{array}$ & $\begin{array}{c}\text { Casi } \\
\text { nunca } \\
\%\end{array}$ & $\begin{array}{c}\text { Casi } \\
\%\end{array}$ & $\begin{array}{c}\text { siempre } \\
\%\end{array}$ \\
\hline $\begin{array}{l}\text { 24. Alguna vez he hablado con mi grupo de } \\
\text { amigos acerca de la discapacidad. }\end{array}$ & 32.8 & 35.7 & 18.3 & 13.1 \\
$\begin{array}{l}\text { 25. He asistido a charlas sobre discapacidad } \\
\text { en mi centro educativo. }\end{array}$ & 51.1 & 23.2 & 14.1 & 11.6 \\
$\begin{array}{l}\text { 26. He leído libros o he visto películas cu- } \\
\text { yos protagonistas eran personas con disca- } \\
\text { pacidad. }\end{array}$ & 17.9 & 29.9 & 27.7 & 24.5 \\
\hline
\end{tabular}


Derechos de las personas con discapacidad

La Tabla 9 determina que el $72.7 \%$ de los participantes está totalmente de acuerdo en que las personas con diversidad funcional puedan tener las mismas oportunidades a nivel escolar, social y laboral que el resto de la sociedad y el $40.1 \%$ considera que la igualdad se produce actualmente. Además, el $69.3 \%$ muestra disconformidad con que estas personas deban relacionarse únicamente con personas con discapacidad.

Tabla 9

Estadísticos descriptivos de la dimensión Derechos de las personas con diversidad funcional de acuerdo con la opinión de los estudiantes de ESO

\begin{tabular}{|c|c|c|c|c|}
\hline $\begin{array}{l}\text { Derechos de las personas } \\
\text { con discapacidad }\end{array}$ & $\begin{array}{c}\text { Totalmente } \\
\text { en desacuerdo } \\
\%\end{array}$ & $\begin{array}{c}\text { En } \\
\text { desacuerdo } \\
\%\end{array}$ & $\begin{array}{c}\text { De } \\
\text { acuerdo } \\
\%\end{array}$ & $\begin{array}{c}\text { Totalmente } \\
\text { de acuerdo } \\
\%\end{array}$ \\
\hline $\begin{array}{l}\text { 29. Es importante que estas } \\
\text { personas tengan las mismas } \\
\text { oportunidades escolares, } \\
\text { sociales y laborales que el } \\
\text { resto de personas. }\end{array}$ & 8.4 & 4.8 & 14.1 & 72.7 \\
\hline $\begin{array}{l}\text { 30. Estas personas deberían } \\
\text { relacionarse únicamente } \\
\text { con personas con discapa- } \\
\text { cidad. }\end{array}$ & 69.3 & 19.1 & 2.5 & 9.1 \\
\hline $\begin{array}{l}\text { 42. Estas personas tienen } \\
\text { igualdad de oportunidades } \\
\text { en su día a día. }\end{array}$ & 14.7 & 22.7 & 22.4 & 40.1 \\
\hline
\end{tabular}

\section{Capacidades y habilidades}

La Tabla 10 afirma que muchos participantes están en desacuerdo con la idea de que estos individuos sean menos inteligentes (54.5\%), menos sociables (42.5\%) e incluso menos valiosos (76.5\%). Tampoco creen que actúen o sean diferentes al resto (34.0\%), ni que estos tengan más dificultad para relacionarse con otras personas (35.3\%). Asimismo, un $44.8 \%$ manifiesta que las características de estas personas no les impiden acceder a estudios superiores ni les limita para comprender instrucciones complejas. Sin embargo, el $55.4 \%$ piensa que las personas con diversidad funcional son capaces de hacer las cosas tan bien como el resto. 
Creencias de los estudiantes de Educación Secundaria Obligatoria sobre la diversidad funcional

María Martínez Galiana, Ma Ángeles Gomariz Vicente y Antonia Cascales Martínez

Tabla 10

Estadísticos descriptivos de la dimensión Capacidades y habilidades de las personas con discapacidad a partir de la opinión de los estudiantes de ESO

\begin{tabular}{|c|c|c|c|c|}
\hline $\begin{array}{l}\text { Capacidades y habilidades de } \\
\text { las personas con discapacidad }\end{array}$ & $\begin{array}{c}\text { Totalmente } \\
\text { en } \\
\text { desacuerdo } \\
\%\end{array}$ & $\begin{array}{c}\text { En } \\
\text { desacuerdo } \\
\%\end{array}$ & $\begin{array}{c}\text { De } \\
\text { acuerdo } \\
\%\end{array}$ & $\begin{array}{l}\text { Totalmente } \\
\text { de } \\
\text { acuerdo } \\
\%\end{array}$ \\
\hline $\begin{array}{l}\text { 31. Las personas con discapaci- } \\
\text { dad son menos inteligentes. }\end{array}$ & 54.5 & 31.1 & 6.6 & 7.7 \\
\hline $\begin{array}{l}\text { 32. Las personas con discapaci- } \\
\text { dad son menos sociables. }\end{array}$ & 42.5 & 34.3 & 16.8 & 6.4 \\
\hline $\begin{array}{l}\text { 33. Las personas con discapaci- } \\
\text { dad actúan o son diferentes del } \\
\text { resto de personas. }\end{array}$ & 27.9 & 34.0 & 30.6 & 7.5 \\
\hline $\begin{array}{l}\text { 34. Las personas con discapaci- } \\
\text { dad son menos valiosas. }\end{array}$ & 76.5 & 12.4 & 2.9 & 8.1 \\
\hline $\begin{array}{l}\text { 35. Las personas con discapa- } \\
\text { cidad son capaces de hacer } \\
\text { muchas cosas tan bien como } \\
\text { cualquier otra persona. }\end{array}$ & 9.0 & 10.9 & 24.7 & 55.4 \\
\hline $\begin{array}{l}\text { 36. Las personas con disca- } \\
\text { pacidad tienen dificultad para } \\
\text { relacionarse con otros. }\end{array}$ & 23.1 & 35.3 & 32.8 & 8.8 \\
\hline $\begin{array}{l}\text { 40. La discapacidad les impide } \\
\text { acceder a estudios superiores } \\
\text { (Bachillerato...). }\end{array}$ & 44.8 & 29.4 & 15.8 & 10.0 \\
\hline $\begin{array}{l}\text { 41. Estas personas solo siguen } \\
\text { instrucciones simples (haz el } \\
\text { ejercicio, siéntate...). }\end{array}$ & 44.8 & 33.5 & 14.0 & 7.7 \\
\hline
\end{tabular}

Adaptaciones y utilización de recursos para dar respuesta a la atención a la diversidad

La Tabla 11 muestra la creencia exclusiva que confirma que estas personas aprenderían mejor en centros de educación especial (41.9\%), a pesar de que el $41.4 \%$ afirma que su centro educativo cuenta con las herramientas y apoyos necesarios para atender sus condiciones personales. Del mismo modo, el 36.9\% confirma que observa adaptaciones en su ciudad, y el $37.9 \%$ que existen becas y ayudas sociales adecuadas. 
Tabla 11

Estadísticos descriptivos de la dimensión Adaptaciones y utilización de recursos para dar respuesta a la diversidad según la opinión de los estudiantes de ESO

\begin{tabular}{|c|c|c|c|c|}
\hline $\begin{array}{l}\text { Adaptaciones y recursos para } \\
\text { las personas con discapacidad }\end{array}$ & $\begin{array}{c}\text { Totalmente } \\
\text { en } \\
\text { desacuerdo } \\
\%\end{array}$ & $\begin{array}{c}\text { En } \\
\text { desacuerdo } \\
\%\end{array}$ & $\begin{array}{l}\text { De } \\
\text { acuerdo } \\
\%\end{array}$ & $\begin{array}{c}\text { Totalmente } \\
\text { de } \\
\text { acuerdo } \\
\%\end{array}$ \\
\hline $\begin{array}{l}\text { 37. Estas personas aprenden } \\
\text { mejor en centros educativos } \\
\text { especiales. }\end{array}$ & 11.8 & 19.7 & 41.9 & 26.7 \\
\hline $\begin{array}{l}\text { 38. Los alumnos con discapa- } \\
\text { cidad de tu centro cuentan con } \\
\text { los recursos y apoyos necesa- } \\
\text { rios (aulas de apoyo, adaptacio- } \\
\text { nes...). }\end{array}$ & 12.0 & 16.1 & 30.5 & 41.4 \\
\hline $\begin{array}{l}\text { 39. Observo adaptaciones } \\
\text { (rampas, semáforos, medios de } \\
\text { transporte, señales adaptadas...) } \\
\text { en mi ciudad para dar respuesta } \\
\text { a la discapacidad. }\end{array}$ & 11.3 & 20.4 & 36.9 & 31.4 \\
\hline $\begin{array}{l}\text { 43. Existen recursos suficientes } \\
\text { para ayudarles (becas, ayudas } \\
\text { sociales...). }\end{array}$ & 8.6 & 17.2 & 36.3 & 37.9 \\
\hline
\end{tabular}

Objetivo 2: Analizar la influencia de las variables sociodemográficas (género, edad, curso que se está estudiando, tipo de centro al que asiste, relación con la discapacidad) en la opinión que tienen los adolescentes sobre la diversidad funcional.

A continuación, se realizó un análisis comparativo de las opiniones que tienen los estudiantes de entre 12 y 18 años de acuerdo con las variables predictoras que se tuvieron en cuenta, como es el caso de la localidad, el género, la edad, el curso y el tipo de centro al que asisten, así como la relación directa que cada uno tiene con la diversidad funcional o el apoyo educativo recibido.

\section{Localidad}

Al realizar la prueba a de contraste de $U$ Mann-Whitney en cada dimensión en función de la localidad de los participantes, los resultados 
muestran diferencias estadísticamente significativas en la relación con

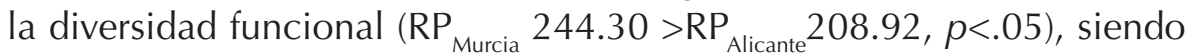
favorable para los chicos encuestados en la Comunidad Autónoma de la Región de Murcia. También en la adquisición de conocimientos sobre esta temática $\left(\mathrm{RP}_{\text {Alicante }} 231.99>\mathrm{RP}_{\text {Murcia }} 196.88, p<.05\right)$, mostrándose esta diferencia a favor de los alumnos encuestados en Alicante.

\section{Género}

En cuanto al género, los datos extraídos de la prueba de contraste de U Mann-Whitney en cada dimensión en función del género de los participantes, revelan diferencias significativas respecto a las dimensiones de la valoración de las capacidades y las habilidades de las personas con discapacidad, $\left(\mathrm{RP}_{\text {Masculino }} 237.51>\mathrm{RP}_{\text {Femenino }} 206.79, p<.05\right)$, a favor del género masculino. También aparecen diferencias en la dimensión sobre las adaptaciones y utilización de recursos $\left(\mathrm{RP}_{\text {Femenino }} 233.19>\mathrm{RP}_{\text {Masculino }}\right.$ 206.84, $p<.05)$, en beneficio del género femenino.

\section{Edad}

Respecto a la edad de los participantes, al aplicar la prueba de Kruskall Wallis se aprecian diferencias estadísticamente significativas en dos dimensiones, tal y como muestra la Tabla 12. Al aplicar la prueba $U$ Mann Whitney se confirman diferencias estadísticamente significativas, tanto en los ítems referidos a los derechos de las personas con discapacidad entre los participantes de 12 y los de 13 años ( $\left(R P_{12 \text { años }} 90.98>\right.$ $\left.\mathrm{RP}_{\text {13años }} 73.35, p<.05\right)$, los de 12 años y los de 14 años $\left(R P_{12 \text { años }} 99.52>\right.$ $\left.\mathrm{RP}_{\text {14años }} 78.69, p<.05\right)$, los de 12 años y los de 15 años $\left(R P_{12 \text { años }} 100.78\right.$

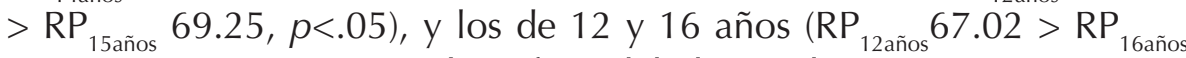
$44.37, p<.05)$, siempre en beneficio del alumnado más pequeño; como en los participantes de entre 14 años respecto a los de $15\left(\mathrm{RP}_{14 \text { años }} 114.27\right.$ $>\mathrm{RP}_{15 \text { años }}$ 97.97, $p<.05$ ), a favor de los primeros.

En cuanto a la dimensión de adaptaciones y utilización de recursos que atienden a la diversidad, cabe destacar diferencias estadísticamente significativas en los alumnos de 12 años respecto a los de $13\left(\mathrm{RP}_{12 \text { años }}\right.$ $\left.89.96>\mathrm{RP}_{\text {13años }} 73.96, p<.05\right), 14$ años $\left(\mathrm{RP}_{12 \text { años }} 96.71>\mathrm{RP}_{\text {14años }} 80.21\right.$,

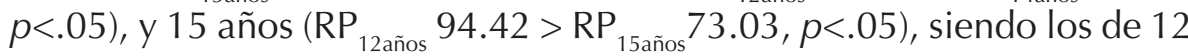
quienes tienen una mejor opinión. Lo mismo ocurre entre el alumnado 
de 15 años respecto del de $17\left(\mathrm{RP}_{17 \text { años }} 74.91>\mathrm{RP}{ }_{15 \text { años }} 56.91, p<.05\right)$, destacando la opinión de los estudiantes de 17 años respecto a esta dimensión, al igual que ocurre entre los estudiantes de 17 y 18 años $\left(\mathrm{RP}_{\text {17аños }} 10.85>\mathrm{RP}_{\text {18аños }} 2.75, p<.05\right)$.

Tabla 12

Prueba de contraste de Kruskal-Wallis en cada dimensión en función de la edad de los participantes

\begin{tabular}{lc}
\hline Dimensiones & Sig. Asintótica \\
\hline Relación con la diversidad funcional & .14 \\
Respeto y tolerancia & .08 \\
Adquisición de conocimientos & .06 \\
Derechos & $.00^{*}$ \\
Capacidades y habilidades & .52 \\
Adaptaciones y utilización de recursos & $.04^{*}$ \\
\hline
\end{tabular}

*Diferencias estadísticamente significativas $(<.05)$

\section{Curso estudiado}

En función del curso estudiado (Tabla 13), se manifiestan diferencias estadísticamente significativas en todas las dimensiones exceptuando la de capacidades y habilidades de las personas con discapacidad. En primer lugar, hay discrepancias entre el alumnado de $1^{\circ}$ de ESO y el de $4^{\mathrm{o}}\left(\mathrm{RP}_{\text {cuarto }} 123.87>\mathrm{RP}_{\text {primero }} 98.51, p<.05\right)$ respecto a la dimensión de la relación con la diversidad funcional. Igualmente, en cuanto al respeto y la tolerancia, existen diferencias estadísticamente significativas entre los estudiantes de $1^{\circ} \mathrm{y}$ los de $2^{\circ}$ de ESO $\left(\mathrm{RP}_{\text {primero }} 136.63>\mathrm{RP}_{\text {segundo }} 117.86\right.$, $p<.05)$, siendo favorable hacia los estudiantes de primero. También entre los que están cursando $2^{\circ}$ y los de $3^{\circ}\left(\mathrm{RP}_{\text {tercero }} 123.95>\mathrm{RP}_{\text {segundo }} 103.63\right.$, $p<.05)$, o entre los de $2^{\circ}$ y $4^{\circ}\left(\mathrm{RP}_{\text {cuarto }} 134.33>\mathrm{RP}_{\text {segundo }} 109.92, p<.05\right)$ en ambos casos en beneficio de los alumnos matriculados en cursos más elevados.

Por último, en cuanto a la adquisición de conocimientos sobre la discapacidad, se contemplan diferencias estadísticamente significativas entre los alumnos de $1^{\circ}$ y los de $2^{\circ}\left(\mathrm{RP}_{\text {segundo }} 137.63>\mathrm{RP}\right.$ primero 113.45 , $p<.05)$ y entre los de $1^{\circ}$ y $4^{\circ}\left(R_{\text {cuarto }} 127.08>R P_{\text {primero }} 95.63, p<.05\right)$, en merced de los adolescentes de $2^{\circ}$ y $4^{\circ}$ de ESO. Por último, hay diferencias entre el estudiantado de $2^{\circ}$ y el de $3^{\circ}\left(R_{\text {segundo }} 118.31>R P_{\text {tercero }} 100.74\right.$, 
Creencias de los estudiantes de Educación Secundaria Obligatoria sobre la diversidad funcional

María Martínez Galiana, Ma Ángeles Gomariz Vicente y Antonia Cascales Martínez

$p<.05)$, con una mayor adquisición de los alumnos de $2^{\circ}$ de ESO; y entre el alumnado de $3^{\circ} 4^{\circ}\left(R_{\text {cuarto }} 106.85>R P_{\text {tercero }} 81.77, p<.05\right)$, siendo los jóvenes de $4^{\circ}$ de ESO quienes han adquirido más aprendizajes sobre esta temática.

Tabla 13

Prueba de contraste de Kruskal-Wallis en cada dimensión en función del curso que están estudiando los participantes

\begin{tabular}{lc}
\hline Dimensiones & Sig. Asintótica \\
\hline Relación con la diversidad funcional & $.03^{*}$ \\
Respeto y tolerancia & $.02^{*}$ \\
Adquisición de conocimientos & $.00^{*}$ \\
Derechos & $.00^{*}$ \\
Capacidades y habilidades & .18 \\
Adaptaciones y utilización de recursos & $.04^{*}$ \\
\hline
\end{tabular}

*Diferencias estadísticamente significativas $(<.05)$

Tipo de centro al que pertenece

En los centros educativos aparecen diferencias estadísticamente significativas entre las diferentes tipologías (Tabla 14); puesto que los centros públicos tienen una mejor visión acerca de la discapacidad, al haber mayor convivencia con estas personas $\left(\mathrm{RP}_{\text {C.Público }} 229.09>\mathrm{RP} \mathrm{C}_{\text {C.Concertado }}\right.$ 184.37, $p<.05)$.

Tabla 14

Prueba de contraste de U Mann Whitney en cada dimensión en función del centro educativo al que acuden los participantes

\begin{tabular}{lc}
\hline Dimensiones & Sig. Asintótica \\
\hline Relación con la diversidad funcional & $.06^{*}$ \\
Respeto y tolerancia & .27 \\
Adquisición de conocimientos & .20 \\
Derechos & .76 \\
Capacidades y habilidades & .37 \\
Adaptaciones y utilización de recursos & .35 \\
\hline
\end{tabular}

*Diferencias estadísticamente significativas (<.05) 
Inclusión de iguales con diversidad funcional

En este caso se observan diferencias estadísticamente significativas en los ítems de la relación con la diversidad funcional $\left(\mathrm{RP}_{\mathrm{Si}} 232.45>\mathrm{RP}_{\mathrm{No}}\right.$ $204.47, p<.05)$ y en la adquisición de conocimientos $\left(R_{S i} 239.39>R P_{N o}\right.$ $193.68, p<.05)$. Los pensamientos y actitudes son más inclusivos al haber compartido aula con iguales con discapacidad, aunque no influye el convivir en la misma aula para aumentar sus derechos $\left(\mathrm{RP}_{\mathrm{No}} 238.32\right.$ $>\mathrm{RP}_{\mathrm{Si}}$ 210.68, $\left.p<.05\right)$. Igualmente, al estar en contacto con individuos con estas condiciones mejora el fomento de los aprendizajes acerca de esta temática.

Asimismo, en la relación con la diversidad funcional está influida por la presencia de algún familiar con discapacidad, siendo más positiva cuando existe algún pariente con estas características $\left(\mathrm{RP}_{\mathrm{Si}} 257.64>\mathrm{RP} \mathrm{N}_{\mathrm{No}}\right.$ 204.40, $p<.05)$.

\section{Apoyo educativo}

En cuanto al apoyo educativo hay diferencias estadísticamente significativas respecto a la dimensión de los derechos de las personas con discapacidad. Existen discrepancias en función de si se recibe apoyo en el aula $\left(R_{\text {Dentrocentro }} 187,15>R P P_{\text {Norecibe }} 154,22, p<.05\right)$, o cuando lo recibe fuera $\left(R_{\text {Fueraaula }} 202,82>R P P_{\text {Norecibe }} 172,87, p<.05\right)$, siempre a favor de recibir cualquier tipo de apoyo, lo que les permite relacionarse con personas con diversidad funcional.

\section{Discusión y conclusiones}

A pesar de que las políticas educativas se llevan promoviendo desde los años 80, las prácticas inclusivas continúan siendo insuficientes según revelan los datos expuestos, dado que los principios de normalización e inclusión no están completamente interiorizados socialmente por los jóvenes de entre 12 y 18 años. Además, tal como se refleja en este estudio, dependiendo de la comunidad en la que se encuentren y de las normativas desarrolladas acerca de esta temática, así como de los centros educativos en que estén escolarizados (públicos o privados), los estudiantes manifiestan unas actitudes más positivas hacia la diversidad, 
lo que fomenta el respeto y la relación con las personas con diversidad funcional.

De acuerdo con Arnáiz (2003), uno de los problemas más relevantes a nivel social es la exclusión de las personas con características personales, sociales o culturales diferentes y ello se refleja en los datos de este estudio. Según Echeita (2014), uno de los aspectos negativos que afectan al alumnado con discapacidad es la colocación de etiquetas que condicionan perjudicialmente sus expectativas y las de las personas que les rodean. En esta línea lo recogen Belmonte, García-Sanz y Morillas (2013) cuando afirman que actualmente la integración de estas personas no es una realidad, puesto que, aunque están empezándose a eliminar los prejuicios existentes hacia ellos, todavía se les considera como individuos incapaces de tomar sus propias decisiones o llevar a cabo tareas por sí solos. Este estudio muestra las escasas relaciones entre las personas con y sin discapacidad, puesto que aunque comparten los mismos espacios, apenas mantienen vínculos afectivos, lo que produce una ausencia de relaciones significativas entre ellos.

Pero, para garantizar una verdadera sociedad inclusiva no solo se debe modificar el entorno físico, sino que se debe cambiar la mentalidad de los individuos que lo conforman (Mampaso, 2004). Por esa razón, Luque y Luque-Rojas (2012) afirman que se deben suprimir los estereotipos y los prejuicios existentes hacia este colectivo, impulsando actitudes más respetuosas a través de la transmisión de valores como la tolerancia o el respeto. Solo así, tal como afirma Echeita (2004), se conseguirá erradicar la discapacidad, por lo que todas las personas contarán con las mismas oportunidades para vivir una vida de calidad.

Otra razón por la que no ha funcionado la inclusión es porque la diversidad funcional continúa considerándose un tema tabú (Talou et al., 2008). Esto queda reflejado en las encuestas, puesto que la mayoría no sabría decir en qué consiste el término de diversidad funcional, ni comprenden que la discapacidad no se compone únicamente de aspectos negativos. Son personas y, por ello, no se trata solo de adaptar sus conocimientos en centros educativos, sino también de favorecer su desarrollo integral como personas a través del aprendizaje de actitudes y habilidades que les permitan desenvolverse socialmente, fomentando las relaciones sociales tanto con iguales como con otros individuos (González-Gil, 2011).

No obstante, este cambio de creencia requiere un cambio en las 
creencias y estereotipos comunitarios desde las primeras edades, en las que se destaquen las posibilidades de acción (González, 2012). Esto queda reflejado en este estudio, puesto que los participantes que se han relacionado en algún momento con personas con discapacidad tienen una mejor visión hacia esta (Aguado et al., 2008; Aguado et al., 2004; Santana, 2010). Sería conveniente promover actividades culturales que permitan la convivencia entre ambos colectivos y que faciliten la eliminación de las etiquetas peyorativas ya que, tal como indica Pineda (2017), al utilizarlas se crea una huella difícil de eliminar que pone en duda sus posibilidades. En esta línea, Muntaner (1998) asegura que no se deben destacar las limitaciones del alumnado, sino potenciar sus capacidades y mejorar su autoconcepto, aspectos que se reflejan en esta investigación dado que los adolescentes participantes con relación directa con la discapacidad tienen una mejor opinión. Solo de esta manera, de acuerdo con Muntaner (1998) y Muratori et al. (2010), se pueden eliminar las barreras sociales y culturales, por lo que serán innecesarias las prácticas educativas inclusivas, puesto que la sociedad será verdaderamente inclusiva.

\section{Referencias}

Aguado, A. L., Alcedo, M. A. y Arias, B.B. (2008). Cambio de actitudes hacia la discapacidad con escolares de primaria. Psicothema, 20(4),697-704.

Aguado, A. L., Flórez, M. A. y Alcedo, M. A. (2004). Programas de cambio de actitudes ante la discapacidad. Psicothema, 76(4), 667-673.

Aguilar Montero, L. A. (1991). El informe Warnock. Recuperado de http://redes-cepalcala.org/inspector/DOCUMENTOS\%20Y\%20LIBROS/ EDUCACION-ESPECIAL/EL\%20 INFORME\%20 WARNOCK\%20-\%20EE.htm.

Arnáiz, P. (2003). Educación Inclusiva: una escuela para todos. Málaga: Ediciones Aljibe.

Arnáiz, P., Guirao, J.M. y Garrido, C.F. (2007). La atención a la Diversidad: del Modelo del Déficit al Modelo Curricular. Archivos Analíticos de Políticas Educativas, 15(23), 1-41. Recuperado de http://www.redalyc.org/articulo.oa?id=275020546023

Bausela, H. E. (2009). Actitudes hacia la discapacidad: estudio de algunas propiedades psicométricas en una muestra de universitarios mexicanos. Revista Iberoamericana de Educación, 49(6), 1-9.

Belmonte, M.L y García-Sanz, M.P. (2013). La escuela de vida, otra mirada a la discapacidad intelectual. Revista Fuentes, 14, 147-170. Recuperado de https://ojs.publius. us.es/ojs/index.php/fuentes/article/view/2357/2176

Belmonte, M.L, García-Sanz M.P. y Morillas, L.R. (2013). ¿Discapacidad o capacidad? 
Creencias de los estudiantes de Educación Secundaria Obligatoria sobre la diversidad funcional

María Martínez Galiana, Ma Ángeles Gomariz Vicente y Antonia Cascales Martínez

Una mirada diferente. Investigación e Innovación Educativa, 996-1004. Recuperado de http://www.uv.es/aidipe/congresos/Actas_XVI_Congreso.pdf

Colectivo loé (2013). Diversidad funcional en España. Hacia la inclusión en igualdad de las personas con discapacidades. Revista Española de Discapacidad, 1(1), 33-46. Recuperado de http://www.cedd.net/redis/index.php/redis/article/ view/35/pdf

Cubo, S., Martín, B. y Ramos, J.L. (2011). Métodos de investigación y análisis de datos en ciencias sociales y de la salud. Madrid: Pirámide.

Dussan, C. P. (2011). Educación inclusiva: un modelo de diversidad humana. Educación y desarrollo social, 5(1), 139-150.

Echeita, G. (2004). ¿Por qué Jorge no puede ir al mismo colegio que su hermano? Un análisis de algunas barreras que dificultan el avance hacia una escuela para todos y con todos. Revista Electrónica Iberoamericana sobre Calidad, Eficacia y Cambio en Educación, 2(2), 31-42. Recuperado de https://dialnet.unirioja.es/servlet/ articulo?codigo $=1065060$

Echeita, G. (2014). Inclusión y exclusión educativa. De nuevo "voz y quebranto". Revista Iberoamericana sobre Calidad, Eficacia y Cambio en Educación, 11(2), 100-118. Recuperado de https://revistas.uam.es/index.php/reice/article/view/2899

Forlin, C., Earle, C., Loreman, T. y Sharma, U. (2011). The Sentiments, Attitudes, and Concerns about Inclusive Education Revised (SACIE-R) Scale for Measuring Pre- Service Teachers' Perceptions about Inclusion. Exceptionality Education International, 21(3), 50-65. Recuperado de https://www.researchgate.net/publication/236029132_The_ Sentiments_Attitudes_and_Concerns_about_Inclusive_Education_Revised_SACIERR_scale_for_measuring_teachers'_perceptions_about_inclusion

García, L.G.A. y Hernández, O.S. (2011). Actitudes hacia la discapacidad de jóvenes y adultos de Chiapas. Universitas Psychologica, 10(3), 817-827.

González-Gil, F. (2011). Inclusión y atención al alumnado con necesidades educativas especiales en España. CEE Participación Educativa 18, 60-78. Recuperado de http:// autismomadrid.es/wp-content/uploads/2011/11/revista18-11.pdf\#page=117

González, M. M. (2012). La legislación educativa y los alumnos con discapacidad: necesidad de actualización. Anuario Facultad de Derecho, 81-105. Recuperado de http:// sid.usal.es/idocs/F8/ART21553/noriega.pdf

Jiménez, A. y Huete, A. (2010). Políticas públicas sobre discapacidad en España. Hacia una perspectiva basada en los derechos. Política y Sociedad, 47(1), 137-152. Recuperado de http://www.um.es/discatif/documentos/PyS/9_jimenez_Huete.pdf

Ley de Integración Social de los Minusválidos (LISMI) (Ley 13/1982, 30 de abril). Boletín Oficial del Estado, núm. 103, 1982, 30 abril.

Ley de Ordenación General del Sistema Educativo (LOGSE) (Ley Orgánica 1/1990, 3 de octubre). Boletín Oficial del Estado, núm. 238, 1990, 4 octubre.

Ley Orgánica de Educación (LOE) (Ley Orgánica 2/2006, 3 de mayo). Boletín Oficial del Estado, núm.106, 2006, 4 mayo.

Ley Orgánica para la Mejora de la Calidad Educativa (LOMCE) (Ley Orgánica 8/2013, 9 de diciembre). Boletín Oficial del Estado, núm. 295, 2013, 10 diciembre.

Luque, D.J. y Luque-Rojas, M.J. (2012). Actitudes de solidaridad y aceptación hacia el 
Creencias de los estudiantes de Educación Secundaria Obligatoria sobre la diversidad funcional María Martínez Galiana, Ma Ángeles Gomariz Vicente y Antonia Cascales Martínez

alumnado con discapacidad. Revista de Educación Inclusiva, 5(2), 25-41. Recuperado dehttps://dialnet.unirioja.es/descarga/articulo/4105443.pdf

Mampaso, J. (2004). Análisis de las preferencias hacia los distintos tipos de discapacidad de los estudiantes de Educación Secundaria Obligatoria. EduPsykhé. Revista de Psicología y Psicopedagogía, 3(2), 179-200. Recuperado de https://dialnet.unirioja.es/ descarga/articulo/1071167.pdf

Muntaner, J.J. (1998). La sociedad ante el deficiente mental: Normalización, integración educativa, inserción social y laboral. Madrid: Narcea.

Muratori, M., Guntín, C. y Delfino, G. (2010). Actitudes de los adolescentes hacia personas con discapacidad: un estudio con alumnos de polimodal en la zona norte del conurbano bonaerense. Revista de Psicología 6(12), 39-56. Recuperado de http:// bibliotecadigital.uca.edu.ar/repositorio/revistas/actitudes-adolescentes-hacia-personas-discapacidad.pdf

O'Dwyer, L. y Bernauer, J. (2014). Quantitative Research for the Qualitative Researcher. Thousand Oaks, California: Sage.

Pineda, P. (2017). Pablo Pineda alerta a docentes valencianos sobre la necesidad de la educación inclusiva. (2017, mayo 24). Recuperado de http://www.levante-emv. com/comunitat-valenciana/2017/05/24/pablo-pineda-alerta-docentes-valencianos/1571212.html

Santana, P. (2010). La actitud hacia la discapacidad de los alumnos de $3^{\circ}$ de ESO del IES Carmen Martín Gaite: análisis a partir de un programa de intervención. Trabajo Fin de Master "Actividad físico deportiva, personas con discapacidad e integración social".

Talou, C.L., Borzi, S.L., Sánchez, M.J., Iglesias, M.C. y Hernández, V. (2008). La educación de los niñas y niñas con diversidad funcional desde la perspectiva de sus pares y docentes. Revista de psicología, (10), 249-260. Recuperado de http://www.memoria. fahce.unlp.edu.ar/art_revistas/pr.4411/pr.4411.pdf 
